Rapid Reviews COVID-19

\title{
Review 2: "Genome sequencing of sewage detects regionally prevalent SARS-CoV-2 variants"
}

Blake Wiedenheft ${ }^{1}$, Anna Nemudraia, Artem Nemudryy ${ }^{1}$ Montana State University Bozeman, Microbiology and Immunology

Published on: Jan 24, 2021

DOI: 10.1162/2e3983f5.109e6e9f

License: Creative Commons Attribution 4.0 International License (CC-BY 4.0). 


\section{$\underline{\text { RR:C19 Evidence Scale rating by reviewer: }}$}

- Strong. The main study claims are very well-justified by the data and analytic methods used. There is little room for doubt that the study produced has very similar results and conclusions as compared with the hypothetical ideal study. The study's main claims should be considered conclusive and actionable without reservation.

$* * * * * * * * * * * * * * * * * * * * * * * * * * * * * * * * * * * * * * * * *$

\section{Review:}

Crits-Christoph et al sequence RNA isolated from wastewater collected at the municipal utility districts in the San Francisco Bay Area. Sequencing results in complete or near-complete genomes for SARS-CoV-2, bocavirus 3, PMMoV, and other plant viruses. They focus on the SARS-CoV-2 sequences and show that the genotypes predominantly correspond to those isolated from clinical genomes in the region. Minor genotypic variants are detected, and the authors suggest that this represents a sensitive way to detect recent introductions to viral lineages before they are detected in clinical samples. Overall, I consider the work to be well- suited for publication in RR:C19.

Questions:

In figure $1 \mathrm{a}$, it is unclear why there are two entries (relative abundances) reported for a single location on some dates, while other locations have a single entry.

Have the authors followed single-nucleotide variants (SNVs) 8083A and 1738T? If these SNVs have appeared in CA clinical samples since July, then it will strengthen author's claim about early detection of recent introduction events.

Minor recommendations:

I think the understanding by a broad readership may benefit from clear definition of "milk of silica."

When authors describe Figure $1 \mathrm{~d}$ in the text "Only samples with RT-qPCR Ct-values $<33$ ( $\sim 25 \mathrm{gc} / \mathrm{uL}$ ) yielded complete consensus genomes" it might be better to talk about total genome copies (as on x-axis on the plot) rather than gc/uL. A dashed vertical line showing the threshold (>1000 genome copies) for achieving complete genome might also help to read the plot. 
Consider commenting on why plant viruses predominate RNA samples isolated using ultrafiltration (panel a) as compared to those from total RNA column and milk of silica samples (panel b). Were both samples amplified using oligo-capture approach to enrich for human respiratory viruses? 